\title{
Adolescent Identification and Academic Achievement: Reporting the Awareness of Similarity to Role Models
}

\author{
Peter Williams ${ }^{l}$
}

Received May 1,1980

Individualized identification grids were administered to 16-18-year-old males $(\mathrm{N}=49)$ in order to chart the complex of identifications they have established with their parents, peers, and school personnel. It was hypothesized that patterns of identification with particular acquaintances would vary as a function of individuals' level of academic achievement. The results of this study indicated that high achievers identified more often with their liked school personnel than did low achievers, and more often with their peers and school personnel than with their parents. Low achievers were shown to most often identify with their mothers and peers. Arguments for viewing identification as a cognitive process based upon reciprocal interpersonal involvement and mediated by situation-specific competence are presented.

\section{INTRODUCTION}

Although identification is a construct firmly entrenched in the personality development literature, the factors contributing to the emergence of this behavior are not entirely clear. Traditional approaches to this phenomenon suggest that identifications occur either as ego defense or as learned response; namely, in reaction to a threat to the person's self-esteem through potential loss of love (Sanford, 1955); in response to a threat of aggression or domination

\footnotetext{
'Student, Educational Psychology Program at The University of Michigan. Current research interests are the interactional effects of skill competence and personal salience of skill areas on the formation of role model identifications in adolescence.
} 
by the more powerful role model (Freud, 1937); as a result of reinforcement based imitation (Bandura, 1965); in response to positive or negative events occuring to the role model (Kagan, 1958); or as an attempt to reproduce bits of the loved and longed-for parent (Mowrer, 1950).

Recent research (Csikszentmilhalyi, 1978) detailing a social-cognitive approach to socialization suggests that identifications are established through the conscious and voluntary focusing of attention by the role model and the identifier on each other. Kagan (1967) previously presented evidence which supports the idea of attentional focusing in identification-based relationships. He reported that individuals direct more attention to role models who are believed to share personological dispositions with the subjects than to models who manifest fewer such dispositions. In a related vein, Rosenkrans (1967) discussed findings which illustrated the most effective role models (in eliciting imitative behavior) were persons with whom observers believed they held the most in common.

The combined effect of individuals' perceptions of similarity to their role models and the attractiveness of the environment which the role model represents can be understood as providing some basis for the formation of situation-specific identifications. In the school setting, Csikszentmihalyi (1978), believes that teacher identification - with the ensuing incorporation of the goals and values the teacher models and represents - are born of certain individuals' understanding that they are able to produce specific behaviors that are at once enjoyable to themselves and pleasing to their teacher. If this speculation is accurate, then identification will occur to the extent that individuals either already share behaviors, interests, and physical characteristics with their role models, or believe that they have the opportunities and potentials for developing such similarities.

McClelland's early work (1961) on the emergence of the achievement motive suggests that parental attitudes can foster school identifications by placing emphasis on academic success and independence from traditional family ties. This push toward academic excellence and individualism has been found to be a pattern of behavior common among the mothers of high achievers (Winterbottom, 1953).

The purpose of the present research was to chart the patterns of identifications that adolescents have formed with their parents, peers, and school personnel in order to test the hypothesis that a positive relationship exists between individuals' level of academic achievement and their patterns of teacher identification. "Identification" was defined as individuals' perceptions of similarity to their acquaintances. It was hypothesized that high academic achievers would perceive themselves to be more similar to their school personnel than would low achievers; and, in turn, low achievers would see themselves as being more similar to their parents and peers than would would high achievers. 


\section{METHOD}

\section{Subjects}

The subjects were 49 adolescent males of predominantly middle class backgrounds, aged 16-18 years. These individuals were volunteers from junior and senior level history and psychology classes at an urban midwestern high school. The personal data collected from these individuals included their cumulative grade point averages, the years of education that each of their parents had completed, and the parents' professional degrees. Testing took place during regular school hours, and all participants were informed of their rights and assured of confidentiality of information.

\section{Technique Development}

The instrument used to obtain a measure of identification was an adaptation of George Kelly's (1955) Role Construct Repertory Test (Reptest). Through the use of indirect verbal self-reports, this technique allows a researcher to make structural sense of individuals' social and psychological worlds. The Reptest taps the constructs individuals use to relate to others by recording how they describe various groups of their acquaintances in regard to their similarities and differences. Identifications are represented by the self-oriented or self-referenced constructs that persons use to describe their relationships with others.

\section{Current Adaptation}

The "identification grid" used in this study was intended to measure subjects' perceptions of similarity to their acquaintances. Individualized instruments were constructed by obtaining lists of the students' school and nonschool acquaintances. The subjects selected from a roster of the school's staff the names of eight persons, both liked and disliked, whom they had come to know well within the past two years. These names, along with those of three liked peers and three disliked peers, were rated by the students on a 7-point like-dislike scale. From this list of school personnel and peers, the names of two liked and two disliked school personnel and one liked and one disliked peer were selected for inclusion on the grid. To these names were added the labels, "my mother," "my father," and "Me (my current self)."

Systematically matched pairs of names and labels were joined with the "Me" tag, and presented to the individuals in protocol booklets. Each iden- 
tification grid consisted of 28 triads of names and labels (of which "Me" was always a part) that offered the subjects opportunities to compare pairs of their acquaintances with each other and in turn with themselves. The subjects were to determine in what important way two of the three persons represented in each comparison group (triad) were similar to each other, and how the third person was different from the other two. This method indicated which individuals the subjects perceived to be the most similar, and on what dimensions these relationships were founded. None of the pairings were repeated, and the students had an equal opportunity to identify with their parents, peers, and school personnel. If subjects chose to identify with certain individuals rather than others, this was taken as an indication that the valence of perceived similarity was stronger toward these individuals than it was toward others. The grid was constructed so that the choice of identifying with one person rather than another at one time did not preclude identifying with that other person at a different time.

\section{Scoring}

Identification scores were obtained by summing the number of times the subjects associated themselves with another person in any of the 28 triads. Frequencies of identifications with school personnel, parents, and peers were tabulated for the individuals, and their response patterns were assessed in relation to their level of academic achievement (grade point average).

\section{RESULTS}

A series of profile analyses followed by pairwise $t$ tests were employed to probe these data. Harris (1975) describes profile analysis as a conservative multivariate analog to the two-way ANOVA (guarding against type II errors), and suggests the use of $t$ tests as a way of performing post hoc comparisons. The sample size was judged adequate for this simple two-factor analysis, and the subject population was segmented into achievement level groups by including all participants with less than a 2.5 grade point average (GPA; $1.0=\mathrm{D}$, $4.0=\mathrm{A}$ ) in a low achievement group, and all participants with a 2.5 or higher GPA in a high achievement group. Divided in this way the groups numbered 23 and 26, respectively (see Table I).

The first profile analysis revealed an interaction between high and low achievers' identification with their parents and liked school personnel, $F(1,47)=$ $8.01, p<0.01$. Further testing of this result revealed that higher achievers identified with their liked school personnel more often than they did with their parents, $t(47)=-2.90, p<0.01$. A second profile analysis suggested that 
Table I. Means and Standard Deviations of Identifications Made with Acquaintances by Achievement Level

\begin{tabular}{lcclll}
\hline & \multicolumn{3}{c}{ Achievement level } \\
\cline { 2 - 3 } \cline { 5 - 6 } Acquaintances & \multicolumn{2}{c}{ High } & & \multicolumn{2}{c}{ Low } \\
\cline { 2 - 3 } \cline { 5 - 6 }$n$ & Mean & $S D$ & & Mean & $S D$ \\
\hline Parents & 4.08 & 2.46 & & 5.65 & 3.33 \\
Peers & 5.73 & 1.84 & & 6.17 & 1.89 \\
Liked school & 6.12 & 3.05 & & 4.35 & 2.36 \\
personnel & \multicolumn{2}{c}{$(N=26)$} & & \multicolumn{2}{c}{$(N=23)$} \\
& & &
\end{tabular}

high and low achievers differed in their frequency of identification with peers and liked school personnel, $F(1,47)=5.10, p<0.05$. The pairwise testing of this outcome showed that low achievers identified with their liked school personnel less often than they did with their peers, $t(47)=2.67, p<0.05$. These results were obtained even though no connection was found between individuals' level of achievement and the ratings they assigned their liked school personnel on the 7-point like-dislike scale $(r=0.17, p>0.1)$.

The final profile analysis indicated a significant relationship between high and low achievers' patterns of identification with their parents and peers, $F(1$, $47)=5.42, p<0.05$. Pairwise probing of this outcome indicated that high achievers identified with their peers significantly more often than they did with their parents, $t(47)=-2.69, p<0.05$.

Correlational analysis indicated that there was a significant negative relationship between parental identification and academic achievement $(r=$ $-0.33, p<0.05)$, with the association being most pronounced for identifications with mothers $(r=-0.34, p<0.02)$, but nonsignificant for fathers, $(r=$ $-0.20, p>0.1)$. Further analysis demonstrated no relationship between students' level of academic achievement and the years of education their parents had completed (fathers' $r=0.15, p>0.1$; mothers' $r=0.14, p>0.1$ ).

\section{DISCUSSION}

The results of this investigation support the speculation that a relationship exists between academic achievement and identification. It is evident that high achievers understand themselves to be more similar to their liked school personnel than do low achievers, and that the former identify with their school personnel more often than with their parents. Because of their close association with academic success and career aspirations, these school acquaintances are probably very salient role models at this point in the high 
achievers' development. After all, the subjects of this study are of the age when the need to be aware of, and concerned with, adequate academic preparation is preeminent. If nothing else, teachers model, or at least discuss and encourage, achievement-oriented values.

It is useful to speculate that high academic achievers have acquired the ability to voluntarily limit the focus of their attention to school-related matters when required, while low academic achievers may not have the same control over their attentional processes. Narrowing the scope of one's attention in order to attain particular goals and to complete tasks is a skill affected by the formation of situation-specific identifications (Csikszentmihalyi, 1978).

One would intuitively expect high achievers to like and therefore identify with school personnel, and low achievers to dislike school personnel and therefore not identify with them. But the data do not support this notion. Although both high and low achievers indicate that they like certain school personnel, high achievers appear to have established some special tie with these individuals which is missing in the relationships formed by low achievers.

The lower level of parental identification demonstrated by high achievers is consistent with Winterbottom's (1953) findings on independence training, and most likely represents the concentration of cognitive effort that high achievers put into the schooling situation. The findings that indicate low achievers maintain fewer identifications with their school personnel while preserving significant ties with their mothers and peers, may also be viewed as related both to academic competence and independence training.

While the subjects of this investigation manifested behaviors that support Winterbottom's contentions on independence training, it should be remembered that these students were predominantly White middle class midwesterners. Significantly different patterns of parental identification might have been found had the subjects been drawn from populations that were matriarchial in structure or non-Western European in lineage.

Although not predicted, the patterns of peer identification manifested by both high and low achievers indicate that neither of these groups seems to have any difficulty getting on the same "wavelength" as their age-mates. This ease of identification can be attributed to being involved in the common experiences which occur during the adolescent stage of development.

The identification process is best understood as involving (1) liking someone and (2) holding something in common with him or her that is more significant than the superficial facade of a coincidental relationship. Competence in academic achievement situations seems to be one sure way for students to gain the positive regard of their teachers; and once the presence of this attentional field is acknowledged and accepted by students, the way to the formation of school-related identification is made clear.

The outcomes of this study suggest that it would be useful to scrutinize identification patterns in order to more fully describe their nature and outline 
their relationship to academic success. The academic setting provides an excellent "natural laboratory" for identification research inasmuch as academic competence and career-goal orientation are dimensions along which all individuals must eventually define and evaluate themselves. Better understanding of the subtleties of the identification process may make it possible to both foster an intrinsic motivation for academic attainment in students, and at the same time inform parents and teachers of the role they play in the formation of individuals' personalities.

\section{ACKNOWLEDGMENTS}

The author would like to thank Hazel Markus, William C. Morse, and Jacquelynne E. Parsons for their support and criticisms in the development of this project.

\section{REFERENCES}

Bandura, A. (1965). Influence of models' reinforcement contingencies on the acquisition of imitative responses. J. Personal. Soc. Psychol. 1: 589-595.

Csikszentmihalyi, M. (1978). Attention and the holistic approach to behavior. In Pope, K. S., and Singer, J. L. (eds.), The Stream of Consciousness: Scientific Investigations into the Flow of Human Experience, Plenum, New York.

Freud, A. (1937). The Ego and Mechanisms of Defense, Hogar th, London.

Harris, R. J. (1975). A Primer of Multivariate Statistics, Academic Press. New York.

Kagan J. (1958). The concept of identification. Psychol. Rev. 65: 296-305.

Kagan, J. (1967). On the need for relativism. Am. Psychologist 22: 131-142.

Kelly, G. A. (1955). The Psychology of Personal Constructs, Norton, New York.

McClelland, D. C. (1961). The Achieving Society, Van Nostrand, Princeton.

Mowrer, O. H. (1950). Learning Theory and Personality Dynamics, Ronald Press, New York.

Rosenkrans, M. A. (1967). Imitation in children as a function of perceived similarity to social model and vicarious reinforcement. J. Personal. Soc. Psychol. 7: 307-315.

Sanford, N. (1955). The dy namics of identification. Psychol. Rev. 62: 106-118.

Winterbottom, M. R. (1953). The relation of childhood training in independence to achievement motivation. Unpublished doctoral dissertation, University of Michigan. 